\title{
Determination of thermal properties of porous building material by infrared thermography
}

by A. Marynowicz*

\begin{abstract}
*Opole University of Technology, Faculty of Civil Engineering and Architecture, Katowicka 48 Str., Opole 45-061, Poland; a.marynowicz@po.opole.pl
\end{abstract}

\begin{abstract}
The implementation of infrared thermography in measurement of the thermal conduction and the heat capacity of the typical porous building material were investigated. The prism-shaped $4 \times 4 \times 4 \mathrm{~cm}$ sample was heated-up for 20 seconds by $660 \mathrm{~nm}$ laser module and the surface temperature changes were recorded continuously by the IR camera. Thereafter, a simple inverse method was applied to determine the researched problem unknowns with the usage of proposed approach. The objective thermal properties, i.e. thermal conductivity and heat capacity, of the clinker brick were estimated. The results were compared with reference measurements performed with Netzsch LFA-427 and DSC404 equipments.
\end{abstract}

\section{Introduction}

The intensive investigations of the internal structure and physical properties of porous building materials are carried out with the use of infrared thermography, including saline transport or moisture content propagation [1, 2, 3, 4]. With a thermovision camera operating principles in mind, it is necessary to produce surface temperature field in the investigated sample to perform the analysis of material behaviour in stationary (single frame) or nonstationary (frames sequence) thermal process. Volumetric heating is relatively easy to perform, however if we want to obtain certain temperature distribution at the sample surface, intended for the further image analysis, a much more complicated method must be applied. We can achieve this goal for example with the use of thin surface heaters [5], a thermal impulse with constant intensity [6], hot air, heating lamps [4] or laser beam [7]. From the practical point of view, the most desirable solution is the simplest one with an easiest accessibility. The intensive development of laser technology enables to produce a required surface heat source in the relatively easy way. Therefore, the small semiconductor laser module was used within performed experiment to determine thermal conductivity and heat capacity of the clinker brick, with the usage of infrared camera.

\section{Computational model}

To achieve the goals featured in the introduction, the optimization-based methodology was developed, starting from the solution of two-dimensional heat transfer problem in the cylindrical coordinates, described by the equation

$$
\frac{\rho_{a p p} c_{p}}{\lambda} \frac{\partial T}{\partial t}=\frac{\partial^{2} T}{\partial r^{2}}+\frac{1}{r} \frac{\partial T}{\partial r}+\frac{\partial^{2} T}{\partial z^{2}}
$$

where $\rho_{a p p}, c_{p}, \lambda, r, z, t$ denotes apparent density, specific heat, thermal conductivity, spatial coordinates and time, respectively. The problem is completed with the initial condition $T=T_{0}$ in the all area occupied by the body (for $t=0$ ), and the boundary conditions

$$
\begin{gathered}
-\left.\lambda \frac{\partial T}{\partial z}\right|_{z=0}=q_{\text {las }}(r)-h_{z}\left(T-T_{e}\right) \quad \text { for } \quad z=0 \\
-\left.\lambda \frac{\partial T}{\partial r}\right|_{r=N}=h_{r}\left(T-T_{e}\right) \text { and }-\left.\lambda \frac{\partial T}{\partial z}\right|_{z=M}=h_{z}\left(T-T_{e}\right) \quad \text { for other edges. }
\end{gathered}
$$

The boundary flux $q_{\text {las }}(r)$, describing the absorbed part of an incident laser beam, has the form

$$
q_{\text {las }}(r)=\frac{2 Q_{a b s}}{\pi R_{e f}^{2}} e^{-\frac{2 r^{2}}{R_{e f}^{2}}} .
$$

The quantities $Q_{a b s}$ and $R_{e f}$ are treated in the similar way, like the ones in the formula describing a laser beam in the technological processes basing on laser treatment of materials [7]. In our case $Q_{a b s}$ denotes the power of the absorbed 


\subsection{1/qirt.2016.080}

laser beam, and $R_{e f}$ is the effective Gaussian $e^{-2}$-radius. The assumption of boundary condition in the form of equation (2) and (4) is justified by the fact of relatively small depth of the transitional zone, in which laser beam transfers into the heat energy. An estimated order of absorption length of low-power diode laser covers the range from several dozens of micrometers for gypsum [8] up to ca. $200 \mu \mathrm{m}$, for the concrete and alumnia-based materials [9]. For the simplification of the computational model, it is assumed, that heat flux is "shifted" by the width of that transitional zone. This approach allows obtaining simpler model of the problem, but, in specific situations, connected with much higher laser power, the volumetric heat sources approach produces more accurate results [9].

The above model introduces also the assumption, that the beam radius has the effective character, i.e. $R_{e f}$ depends on superficial properties of the sample. This is motivated by the high variations of the sample surface roughness, as the consequence of a different internal structure of porous building materials. This may change the range of the laser beam interaction with the material, also in the direction lateral to the surface. The above assumption was confirmed in additional calibration measurements.
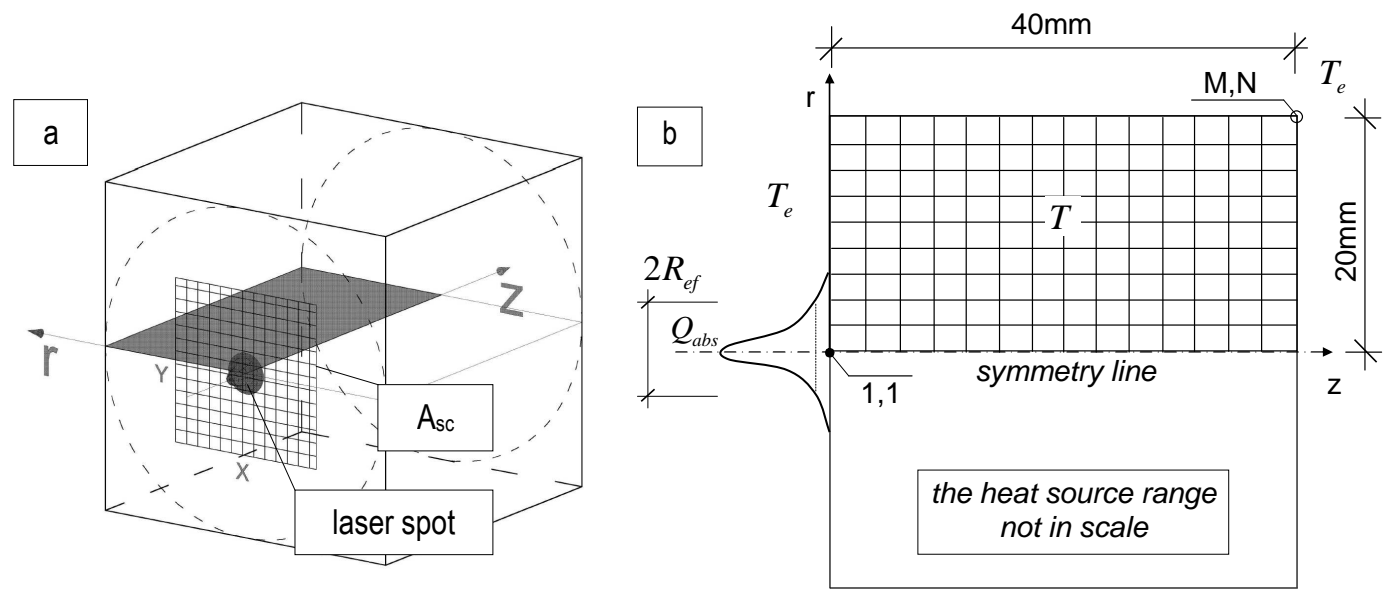

Fig.1. Sample geometry, (a), and it's computational model, (b).

For the later calculations the quantities have values $Q_{a b s}=60 \mathrm{~mW}$ and $R_{e f}=1.6 \mathrm{~mm}$, estimated for the same brick-type material. The heat transfer coefficients were initially assumed to have an equal value, i.e. $h_{r}=h_{z}=7.7 \mathrm{~W} \cdot \mathrm{m}^{-2} \cdot K^{-1}$, what is usually provided for the heat exchange calculations near vertical interior planes, without forced convection. For the estimation of the model sensitivity for changes of heat transfer coefficient variations, the additional calculations have been preformed. After the assumption that convectional flux is negligible in comparison with the laser induced surface heat source, what was possible because $h_{z}\left(T-T_{e}\right) \ll \mathrm{q}_{\text {las }}$ [10], we found, that the differences between the resulting heat conductivities do not exceed $1 \%$. Therefore, the rejection of the convectional part of the boundary conditions (2) and (3) is justified for the future developments of the proposed method.

The unknown parameters, i.e. $\mathbf{u}=\left[\lambda, C_{p}=\rho_{a p p} c_{p}\right]$, were estimated using the direct search method for minimalization of objective function $F(\mathbf{u})$ given in the form

$$
\mathbf{u}=\arg \min _{\mathbf{u}} F(\mathbf{u})=\sum_{i=1}^{n}\left[\left.Y_{i}\left(r_{i}, t\right)\right|_{z=0}-\left.T_{i}\left(\mathbf{u}, r_{i}, t\right)\right|_{z=0}\right]^{2},
$$

where $Y_{i}\left(r_{i}, t\right)$ denotes the vector of temperature measured at the surface $(\mathrm{z}=0)$ at the $\mathrm{i}$-th point $r$ and the time $t$. The vector of temperature $T_{i}\left(\mathbf{u}, r_{i}, t\right)$ was calculated at the same points, with the specified set of parameters $\mathbf{u}$. The values of $T_{i}$ elements were calculated numerically from equation (1), with boundary conditions (2) and (3), by the means of twodimensional Finite Difference Method (FDM), according to the Peaceman-Rachford implicit ADI scheme. The ADI technique is based on the unconditionally stable, implicit solution of the problem in separate directions. The analysed domain has been divided into equally sized elements in both directions (figure 1a). The elements have the dimensions of $0.305 \times 0.305 \mathrm{~mm}$, what corresponds to the size of the area seen by the one pixel of IR camera. For the further analysis, only the part of sample surface, denoted $\mathrm{A}_{\mathrm{sc}}$ (figure 1a), was taken into account, due to relatively small laser spot range.

The simplification of the sample geometry model (figure 1b), described above, was justified by the fact that the time consuming 3D analysis of the same domain led to the very similar results. For the comparison of the two models, the 3D finite difference scheme was developed. The differences in the resulting surface temperature between 2D and 3D models were negligible. Therefore it has been assumed that the proposed simplification is suitable for a further analysis. 


\subsection{1/qirt.2016.080}

\section{Experimental technique}

In order to perform temperature measurements, during sample surface-heating-up process by the laser beam, the experimental assembly was build, as shown in figure 2. The sample was placed on the wooden stand, equipped with graphite-coated shield to reduce beam reflections effects. As the heat source, we used the low-power laser diode module (Mitsubishi ML101J27, 660nm, max. 150 mW CW), together with anamorphic prism pair (Thorlabs), for the beam shape correction. As the temperature recording device, the VigoCAM v50 infrared camera (VIGO System, NETD=0.08K, spectral range $8 \div 14 \mu \mathrm{m}$ ) was used. The assembly was completed with a stable laser power supply unit and a PC computer. The measurements were taken within room conditions. Before the measurements the sample was stored in the room temperature, with the air humidity about $\mathrm{RH}=65 \%$.

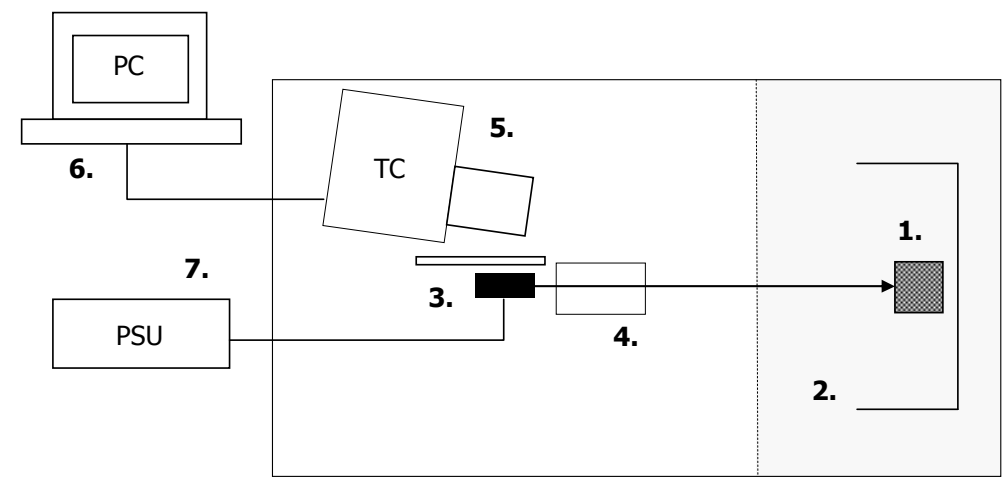

Fig.2. Test assembly scheme: 1- sample on a stand, 2- shield, 3- laser module, 4- correction prisms, 5infrared camera, 6-PC computer, 7- power supply unit.

The accuracy of the infrared temperature measurements of physically heterogeneous materials is affected with numerous errors, arising from different emissivities of individual fragments of the analysed area. The simple auxiliary measurement was performed to evaluate the aforementioned effects. In figure $3 a$, the surface structure of analyzed material is shown in the visible range, with numerous aggregate intrusions clearly visible. Furthermore, figure $3 \mathrm{~b}$ contains the images of the same sample in infrared range, where we can see the effects of intrusions on temperature readings. The image was taken during uniform cooling, after long-time conditioning in the constant temperature $T=70^{\circ} \mathrm{C}$. The quality of temperature readings (see figure 4) allows assuming, that "thermal noise" shown in figure $3 \mathrm{~b}$ is of minor significance for analyzed material.
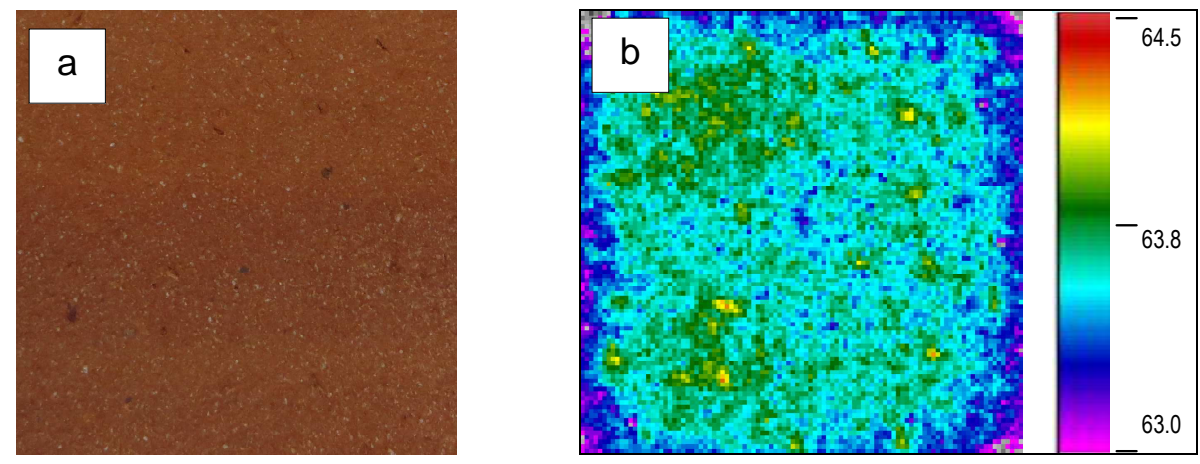

Fig.3. Surface image taken by a digital camera (a) and corresponding infrared image (b) of the brick sample

\section{Results}

The sequence of 20 frames, from 1 to 20 s with 1 s step, was recorded during the experiment. Line $L$ (see figure 4a), close to the spot's horizontal axis was chosen, as the most appropriate for further analysis. The reduction of the measurement time to $20 \mathrm{~s}$ was sufficient due to fast temperature stabilization in the center of the spot. It also allowed for elimination of the drift of bolometric sensing matrix of the IR camera.

As the result of calculations of the mean squared error function values, the minimum of $F(\mathbf{u})$ was found, with $\mathbf{u}=\left[\lambda, C_{p}\right]$, for the specified heating times $t_{i}=1,2 \ldots 20 \mathrm{~s}$. Figure 5 shows, that the solution is correctly determined due to single minimum of $F(\mathbf{u})$. 

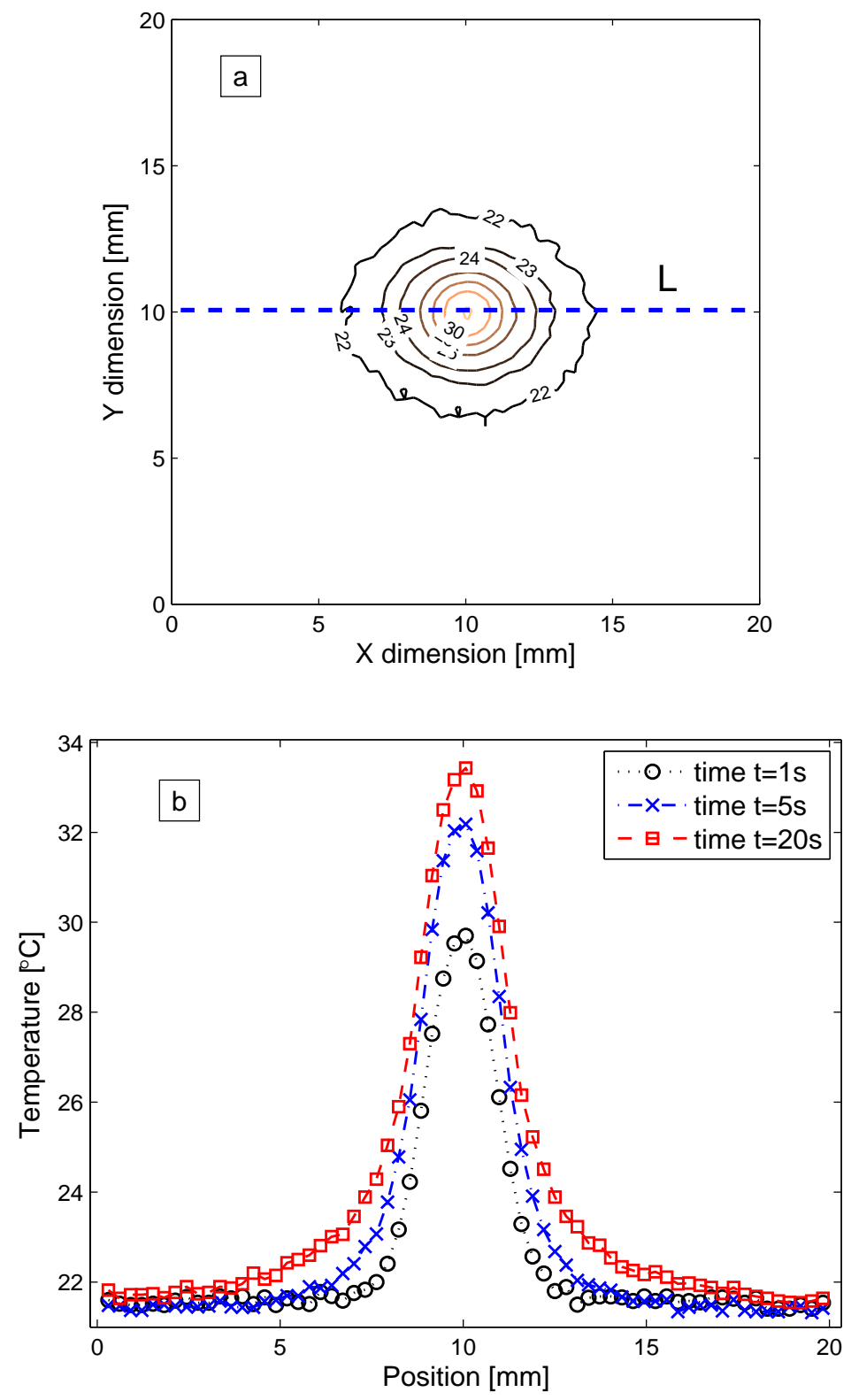

Fig. 4. Temperature isolines at the surface $A_{s c}$ of the sample after 5 s of heating up (a) and temperature at line $L$ in the selected moments of process (b).

In figures 6 and 7 we can see the calculated results for $\lambda$ and $C_{p}$, for all the analyzed times. To calculate the mean value, $\bar{C}_{p}$, the estimated $C_{p}$ for the time $4-15$ s were taken. The results for both searched parameters were compared with the reference measurements with very good agreement for thermal conductivity (figure 6), whereas thermal capacity revealed distinctly bigger results dispersion (figure 7). The value of $C_{p \text {,ref,min }}$ was obtained from measurement performed with differential scanning calorimetry method (Netzsch DSC 404), whereas $C_{p \text {,ref,max }}$ was measured by the laser-flash method (Netzsch LFA 427). Both reference values for the heat conductivity, i.e. $\lambda_{\text {ref,max }}$ and $\lambda_{\text {ref,min }}$, were measured by the laser-flash method. 


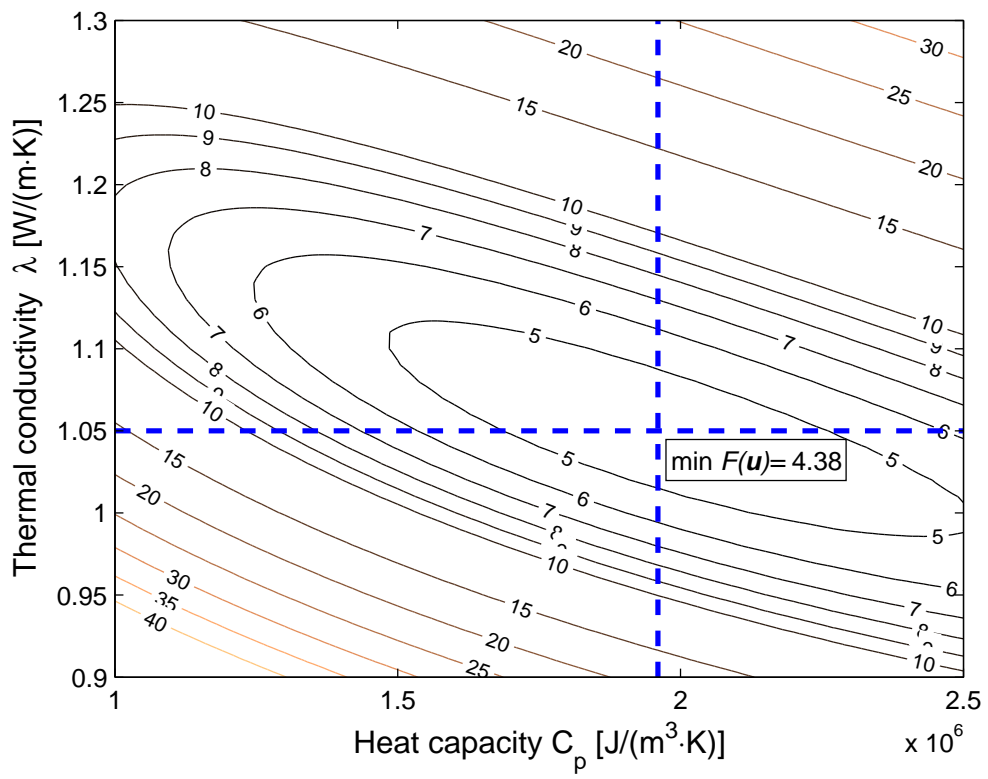

Fig. 5. Error function plot of $F(\mathbf{u})$ for $t=5 s$

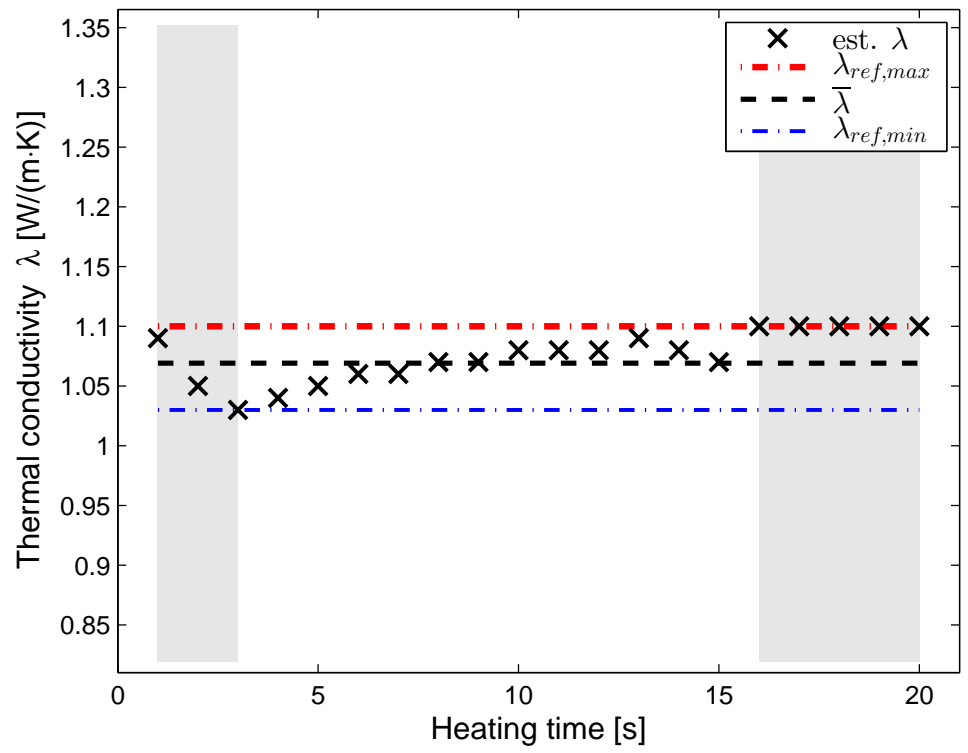

Fig. 6. Estimated thermal conductivity of the clinker brick with reference values 


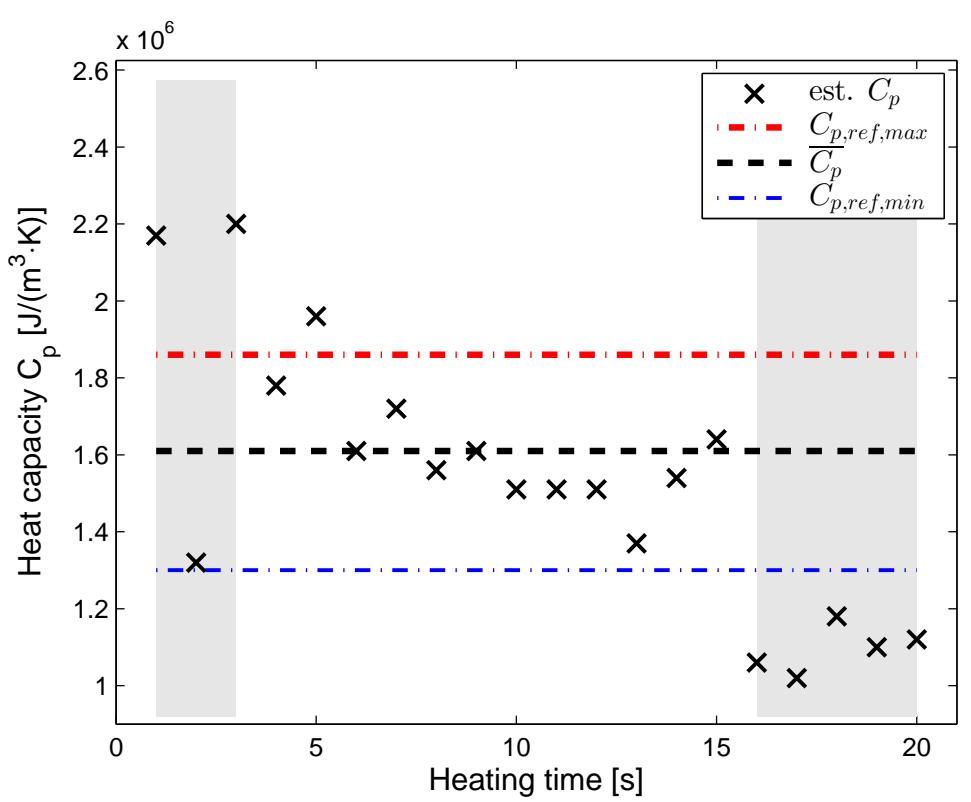

Fig. 7. Estimated heat capacity of the clinker brick with reference values

\section{Conclusions}

After analysis of resulting error function $F(\mathbf{u})$ distributions, we can conclude that the proposed approach gives very good estimation of thermal conductivity for all the time steps (see figure 6), what has been confirmed by the independent reference measurement. The next results, concerning the estimation of the heat capacity, do not give the unique answer. Some of the results show unexplainable, by that moment, variations from the mean value (the bold dashed line in figure 7). After rejecting of these results, we have found that resulting heat capacity range covers one obtained from the reference measurement, but only for the selected time period. Despite of this, we can conclude that the proposed method gives reliable results and may be utilized in the next research. All the fluctuations, lying in the shadowed region in the resulting plot in the figure 6 and 7, need further investigations. They may arise from measurement uncertainties, difficult for an evaluation, for example concerning the time measurements accuracy of IR camera.

\section{REFERENCES}

[1] Barreira E., de Freitas V.P.: Evaluation of building materials using infrared thermography, Construction and Building Materials 21 (2007), pp. 218-224

[2] Bison P., Bortolin A., Cadelano G., Ferrarini G., Grinzato E.: Comparison of some thermographic techniques applied to thermal properties characterization of porous materials, 11th International Conference on Quantitative InfraRed Thermography QIRT Naples, Italy, 2012.

[3] Gayo E., de Frutos J., Palomo A., Massa S.: A mathematical model simulating the evaporation processes in building materials: Experimental checking through infrared thermography, Building and Environment 31 (1996), pp. 469-475

[4] Grinzato E., Vavilov V., Kauppinen T.: Quantitative infrared thermography in buildings, Energy and Buildings 29 (1998), pp. 1-9.

[5] Zmywaczyk J., Madura H., Koniorczyk P., Dabrowski M.: Estimation of thermophysical properties by an inverse method with experimentally determined heating region of a thin-layer heater, Infrared Physics and Technology 49 (2007), pp. 277-280

[6] Perkowski Z.: A thermal diffusivity determination method using thermography: Theoretical background and verification, International Journal of Heat and Mass Transfer 54 (2011), pp. 2126-2135

[7] Bison P.; Cernuschi, F., Grinzato, E., Marinetti, S., Robba, D.: Ageing evaluation of thermal barrier coatings by thermal diffusivity, Infrared Physics and Technology 49 (2007), pp. 286-291

[8] Wojtatowicz T. (ed.): Investigations of phase transitions in surface layer of porous body with use of laser irradiation (in Polish), Politechnika Łódzka, Łódź, 2000

[9] Li J.F., Li. L., Stott F.H: Comparison of volumetric and surface heating sources in the modeling of laser melting of ceramic materials, International Journal of Heat and Mass Transfer 47 (2004), pp. 1159-1174

[10] Dowden J. (ed.): The theory of laser materials processing, Canopus Academic Publishing Ltd, 2009 